\title{
Le designer de jeux vidéo comme agent d'évolution à l'ère du jeu casual
}

\section{Laureline Chiapello}

\section{(2) OpenEdition}

\section{Journals}

Édition électronique

URL : http://journals.openedition.org/sdj/474

DOI : $10.4000 /$ sdj. 474

ISSN : 2269-2657

Éditeur

Laboratoire EXPERICE - Centre de Recherche Interuniversitaire Expérience Ressources Culturelles Education

\section{Référence électronique}

Laureline Chiapello, "Le designer de jeux vidéo comme agent d'évolution à l'ère du jeu casual », Sciences du jeu [En ligne], 4 | 2015, mis en ligne le 04 octobre 2015, consulté le 06 avril 2021. URL: http://journals.openedition.org/sdj/474 ; DOl : https://doi.org/10.4000/sdj.474

Ce document a été généré automatiquement le 6 avril 2021.

\section{(c) (i) (9)}

La revue Sciences du jeu est mise à disposition selon les termes de la Licence Creative Commons Attribution - Pas d'Utilisation Commerciale - Pas de Modification 4.0 International. 


\title{
Le designer de jeux vidéo comme agent d'évolution à l'ère du jeu
} casual

\author{
Laureline Chiapello
}

1 Le lien entre les jeux vidéo et leurs créateurs est étonnamment ambigu. Les grands succès vidéoludiques sont souvent associés à la personnalité d'un designer de jeux, et l'évolution des jeux vidéo paraît alors reposer sur le génie de quelques créateurs inspirés (Donovan, 2010). Pourtant, dans la littérature scientifique, l'évolution des genres et des styles semble se réaliser de façon désincarnée. L'étude des mécaniques, des motifs ou des thèmes apparaissant dans les œuvres vidéoludiques n'est que rarement mise en relation avec le processus de design qui a pu les produire (Djaouti, Alvarez et Jessel, 2010). Il existe pourtant un designer - si ce n'est toute une équipe de designers - pour chaque jeu, et il demeure paradoxal que son rôle ne soit pas mieux documenté. Les études sur l'évolution du jeu casual ${ }^{1}$ font partie de cette mouvance étonnante. Les jeux dits «casual » ont été définis comme étant «faciles à apprendre, simples à jouer (easy to learn, simple to play) $»^{2}$ (Kuittinen et al., 2007, p. 106), ou encore comme des « jeux pour tous » (Kuittinen et al., 2007), accessibles à un public de non-initiés. Ils ont rompu avec la tradition voulant que les nouveaux jeux vidéo s'adressent à une audience toujours plus expérimentée - labellisée hardcore - et sont venus transformer le paysage vidéoludique. Or, dans les écrits académiques, ces jeux semblent apparus d'eux-mêmes, leurs caractéristiques étant analysées comme le résultat de mutations spontanées. L'étude originale sur laquelle s'appuie cet article a choisi ce constat comme point de départ, et a essayé de donner une nouvelle vision du jeu casual en utilisant le savoir professionnel des designers de jeux vidéo (Chiapello, 2012a). Il est apparu que le rôle du designer s'est trouvé fortement remis en question lors de la production de jeux casual. Les nouveautés constatées au niveau de la culture du jeu ont eu des répercussions sur la perception du rôle des designers de jeux vidéo.

2 Cet article souhaite revenir sur l'expérience vécue par les designers de jeux lors de projets casual. En documentant cette expérience, il est possible de mettre en évidence 
les stéréotypes attachés au rôle du designer de jeux. Ces stéréotypes peuvent limiter notre compréhension de l'évolution des jeux, car ils occultent certaines activités des designers. Reconstruire le rôle du designer de jeux dans toute sa richesse semble alors nécessaire pour comprendre en quoi il est un agent d'évolution des jeux.

Dans un premier temps, une recension des écrits permettra de présenter les évolutions de la culture du jeu suscitées par le jeu casual. Ces évolutions ne prennent pas en considération les changements rencontrés dans les pratiques des designers de jeux: cette absence sera l'occasion de rappeler à quel point cette profession clé du jeu vidéo reste peu étudiée. Ce manque d'étude renvoie directement aux difficultés d'accès à l'information, et l'approche méthodologique utilisée, qui a permis de contourner un certain nombre de barrières, sera exposée en détail. Enfin, la présentation des résultats permettra de lier les problématiques apparues avec le jeu casual à une réflexion sur le rôle des designers de jeux vidéo. Les designers ne sont pas simplement des commentateurs passifs devant l'histoire vidéoludique, mais bien des agents d'évolution, et l'histoire des jeux ne peut s'écrire sans considérer ceux qui sont à l'origine de la création des jeux.

\section{Culture du jeu casual : une évolution désincarnée}

4 L'apparition de nombreux jeux vidéo dits « casual » dans le paysage vidéoludique a été appréhendée par les chercheurs comme un phénomène à la fois marquant et complexe. Si le terme "casual» reste aujourd'hui mal défini, plusieurs travaux académiques d'importance ont permis de mieux circonscrire le «phénomène casual ». Ainsi, l'idée d'évolution est au cœur des discussions, et plusieurs chercheurs ont proposé leur propre explication des changements constatés dans la culture du jeu vidéo à l'ère du jeu casual. Cependant, les recherches sont encore discordantes, et restent avant tout centrées sur les jeux et les joueurs; les modalités de création des jeux, en amont de la réception, demeurent peu explorées: elles constituent pourtant une piste d'investigation prometteuse.

\section{Retour sur l'évolution de la culture du jeu entrainée par le phénomène du jeu casual}

5 La vision la plus connue de l'évolution des jeux casual est peut-être celle de «révolution» que Jesper Juul a présentée dans son ouvrage A Casual Revolution Reinventing Video Games and Their Players (2009). L'auteur estime qu'il est nécessaire d'analyser la relation entre les jeux vidéo et les joueurs pour comprendre le jeu casual. Il indique que de ce point de vue, le jeu casual est un phénomène entièrement nouveau: "La révolution casual propose aux joueurs une nouvelle façon de prendre part aux jeux " (Juul, 2009, p. 22). Ces idées de "réinvention », et de "révolution » viennent de l'opposition que le terme « casual » implique face au terme « hardcore»: durant les années 80 et 90, les jeux vidéo semblent uniquement dirigés vers ce public hardcore. Selon Juul, le stéréotype veut que les joueurs hardcore soient principalement des hommes, aimant les thèmes reliés à la guerre et à la science-fiction, ayant joué à un grand nombre de jeux et prêts à investir de vastes ressources, en terme de temps et d'argent, dans leurs activités vidéoludiques. Ils constituent une sorte de "tribu » dont les goûts ont longtemps dicté le contenu de la majorité du paysage vidéoludique. Le jeu 
casual est venu bouleverser leurs habitudes en proposant des jeux moins exigeants en termes de temps, plus accessibles, et attirant un public plus large.

D'un autre côté, Juul nous informe aussi du fait que les premiers jeux vidéo semblaient avoir la même vocation que le jeu casual d'aujourd'hui : celle d'être des divertissements pour tous. En effet, lors de la naissance des jeux vidéo, ces derniers étaient considérés comme un loisir grand public, accepté par tous les âges. Juul n'explique pas véritablement quels sont les facteurs qui ont provoqué la transformation du paysage vidéoludique en un territoire hardcore, mais il note que la fracture est remarquable dès le début des années quatre-vingt. Dans ces conditions, les jeux casual, qui apparaissent massivement au début des années 2000, ne constituent-ils pas plutôt un retour aux sources qu'une révolution? Dans les deux cas, pour Jesper Juul, une période de rupture s'est opérée entre les débuts du jeu vidéo et le jeu casual tel qu'on le connait aujourd'hui.

7 La chercheuse finlandaise Annakaisa Kultima (2009) choisit une autre voie et s'attache à présenter le jeu casual comme une normalisation de la culture du jeu vidéo (p.60). La vision de Kultima témoigne d'une approche plus distanciée que celle de Juul, et s'intéresse à la façon dont le jeu casual s'inscrit dans la société en général, et non uniquement dans les milieux vidéoludiques. Elle relève que les jeux vidéo se sont insérés peu à peu dans nos sociétés, et la tendance casual serait alors le résultat de cette intégration graduelle. Pour Kultima, la transformation de la culture du jeu comporte deux caractéristiques principales: l'arrivée de nouveaux groupes d'utilisateurs hétérogènes et l'avènement du jeu comme une activité secondaire ou utilisée de façon instrumentale. Elle constate une interpénétration entre les activités quotidiennes et les jeux vidéo: il est possible de jouer tout en faisant autre chose, comme manger ou répondre au téléphone. Cet aspect a été lui aussi souligné par Juul qui rapporte que dans son sondage auprès des joueurs : « répondre au téléphone a été rapporté comme étant la source d'interruption la plus courante» (Juul, 2009, p. 37) ; mais pour Kultima, ces transformations ne sont pas étonnantes : elles coïncident avec la "numérisation » de notre environnement, où le monde physique et le monde numérique se mélangent continuellement. Le jeu casual est donc le résultat de ces transformations, qui se sont réalisées progressivement et sans fracas.

Dans son livre Casual game Design Designing: Play for the Gamer in All of Us (2010), Gregory Trefry propose lui aussi une vision du jeu casual comme étant une évolution «tranquille »: en retraçant l'émergence de la tendance casual à travers trois jeux - Le Solitaire, Bejeweled et Wii Sport - il tisse des liens entre les mécaniques de jeux allant des années quatre-vingt-dix jusqu'à 2010. Il définit les caractéristiques des jeux casual comme un héritage des générations de jeux passées.

9 Révolution d'un côté, normalisation de l'autre, la façon dont s'inscrit le jeu casual dans le paysage vidéoludique semble dépendre de la sphère de référence utilisée pour l'analyse (le milieu vidéoludique ou la société son ensemble). Cependant, ces constats concernent principalement l'évolution des caractéristiques des jeux ainsi que celles des joueurs, et les conclusions sont assez succinctes en ce qui concerne les développeurs de jeux vidéo et plus particulièrement les designers. Juul, Kultima et Trefry ont pris soin d'analyser des jeux, d'interroger des joueurs et de consulter des développeurs de jeux, mais l'expérience de ces derniers n'a pas été analysée. Comment la création de jeux casual est-elle vécue par les designers de jeux vidéo? Leur rôle a-t-il changé ? Leurs 
manières de faire ont-elles été remises en question par ce nouveau style de jeux ? Existe-t-il des liens entre l'évolution des jeux et l'évolution des pratiques de création?

\section{Manque de données sur l'expérience des designers de jeux} épanouissement des études du jeu, le rôle des designers de jeux vidéo reste difficile à appréhender, et demeure souvent idéalisé. Comme l'explique O'Donnell, le designer de jeux vidéo est trop souvent vu comme une rock star de la création : «Beaucoup de gens imaginent que le développement de jeux vidéo s'apparente au fait de jouer à des jeux et que tous les développeurs sont les membres célèbres d'un "groupe " de développement " (O'Donnell, 2014, p. 40). Ce mythe est entretenu par l'industrie ellemême et il semble difficile à éviter pour le non-initié. Ce manque de connaissance des activités des designers de jeu a été souligné dans la littérature vidéoludique à plusieurs reprises : « Même si cette affirmation peut sembler évidente, elle implique que l'activité de design demeure négligée » (Kuittinen et Holopainen, 2009, p. 7), ou encore : «Les livres de design [de jeux] se focalisent principalement sur la cible du processus de design : les jeux eux-mêmes. La pratique consistant à créer des jeux en tant qu'activité de design n'est pas traitée. » (Kultima, Köönikkä et Karvinen, 2011, p. 34). Les écrits en études du jeu s'attardent bien souvent sur «le design du jeu», c'est-à-dire les caractéristiques formelles de l'œuvre vidéoludique, mais rares sont ceux qui détaillent le "design de jeux », c'est-à-dire la pratique des designers de jeux, leurs activités quotidiennes. Casey O'Donnell adresse la question suivante aux chercheurs : «Qu'en est-il des gens qui créent l'objet que vous étudiez ?» (O’Donnell, 2014, p. 9).

11 En dehors de la sphère des études du jeu, différents champs d'études se sont intéressés aux pratiques professionnelles en lien avec le jeu vidéo. Dans le domaine du management, on remarque les travaux de Laurent Simon en collaboration avec Ubisoft. Cet auteur s'est intéressé à la gestion de la création ; s'il s'agit bien d'étudier la création de jeux vidéo, le design et les activités des designers de jeux ne sont pas le point focal de ces recherches, plutôt orientées vers ladite gestion. Du côté de l'étude des sciences et technologies (en anglais Science and Technology Studies, souvent abrégé en STS), on note les travaux ethnographiques de Natasha Schüll (2012), qui s'est intéressée au design des jeux électroniques d'argent et de hasard à Las Vegas, et ceux de Thomas Malaby (2009), qui a intégré la compagnie Linden Labs, à l'origine du monde virtuel Second Life. Encore une fois, ces études sont connexes au jeu vidéo, mais leur champ d'investigation ne coïncide pas totalement avec celui-ci. Le livre de Casey O'Donnell, Developer's Dilemma the Secret World of Videogame Creators (2014) est bel et bien une ethnographie d'une entreprise de jeux vidéo et semble constituer l'un des témoignages les plus aboutis sur l'industrie vidéoludique. Par contre, l'activité de design de jeux y est analysée au regard des STS et du travail dans les industries créatives, et l'ouvrage ne porte pas uniquement sur les designers de jeux mais bien sur tous les participants au développement d'un jeu, des programmeurs aux artistes en passant par les producteurs. Ce livre demeure toutefois une nouvelle référence pour appréhender l'industrie vidéoludique et permet de porter un regard plus riche sur les résultats de la présente étude.

Il est aussi possible de compléter notre vision des designers de jeux vidéo à l'aide de la littérature non scientifique. Tout d'abord, il existe des ouvrages consacrés à des 
designers de jeux vidéo dont les œuvres ont connu un succès auprès du public. En français, la collection Les grands noms du jeu vidéo de l'éditeur Pix'n Love propose ainsi un ensemble de biographies officielles de designers tels que Gunpei Yokoi (Makino et Gorges, 2010) ou Eric Chahi (Ichbiah, 2013). Ces ouvrages étant approuvés par le biographié, leur ton est généralement positif. En anglais, une nouvelle collection aux objectifs similaires vient de voir le jour chez Bloomsbury Press, baptisée The Influential Video Game Designers. Selon ses éditeurs, la collection vient précisément remplir le vide que nous évoquions au début de cet article : l'absence de documentation sur les créateurs de jeux vidéo. L'angle choisi est volontairement celui du " designer-auteur ", ce qui rejoint l'idée de fascination exercée par ces créateurs décrite par O'Donnell. D'autres sources peuvent néanmoins fournir des visions plus nuancées, voire négatives. Ainsi, l'éditeur Pix'n Love a aussi publié des ouvrages non officiels, tels que la biographie de Shigeru Miyamoto réalisée par William Audureau (2014). Ce dernier déclare avoir tenté de briser l'image du designer solitaire et "porté par la grâce " (Verlet, 2014). De plus, les sites Web consacrés à la pratique professionnelle, tels que Gamasutra.com ou Develop-online.net, donnent parfois la parole à des designers afin qu'ils expriment leur point de vue sur leur industrie. Ces prises de paroles révèlent souvent les difficultés liées à la profession, comme cela a été le cas récemment avec l'article « Death of the game designer » de Greg Wondra (2015). L'auteur y expose les transformations qu'il a pu constater dans l'industrie, en particulier la tendance à la surspécialisation des designers et l'extrême dévouement des employés exigé par les compagnies vidéoludiques. Dans cette description, le designer n'apparaît plus comme une star mais comme un individu broyé par un système économique impitoyable. Bien que cet ensemble de textes ne soit pas académique, il permet de venir enrichir notre problématique : l'expérience des designers de jeux est variée et les analyses qui en sont faites nécessitent d'être mises en contexte.

Cet article n'est pas le fruit d'une démarche anthropologique, mais d'une recherche en design. Le but premier de l'étude n'était nullement de glorifier le designer comme auteur, mais de mieux comprendre ce qui définissait un jeu casual, en s'appuyant sur les savoirs professionnels des designers de jeux vidéo. Dans cette optique, le cadre théorique qui a été choisi est celui du praticien réflexif de Donald Schön (1983). Selon ce dernier, les professionnels ne sont pas les applicateurs aveugles d'un savoir qui leur a été délivré en amont de leur tâche. Il existe aussi un savoir tacite, acquis par la pratique. Ce savoir se manifeste dans l'action, et demeure implicite dans les tâches routinières du praticien. Cependant, lorsqu'une situation inattendue se présente - par exemple lors de la création d'un nouveau style de jeux - le savoir en action du praticien peut être mis en défaut, et une réflexion sur la procédure à suivre s'enclenche alors. Cette réflexion se fait en action : le praticien modifie la situation problématique, évalue les conséquences de ses actions, et peut ainsi poursuivre son activité dans la bonne direction. De cette réflexion en action naît un nouveau savoir, qui n'est que rarement verbalisé, mais qui existe malgré tout. Les designers de jeux sont ainsi les détenteurs d'un savoir professionnel sur le jeu casual qui leur vient de leurs expériences sur des projets de jeux casual.

14 Le savoir professionnel ne peut se développer que s'il est soutenu par ce que Schön nomme les «constantes». Il s'agit d'un ensemble d'éléments de référence sur lesquels le praticien s'appuie dans sa réflexion en action : un langage particulier, des outils, des valeurs, des théories, et, la plus importante pour cet article, une certaine vision de leur rôle. Le rôle a une fonction d'encadrement, de guide, et permet au professionnel 
d'agir en toute confiance: "Les différentes façons de définir un rôle permettent de déterminer quel savoir est vu comme utile en pratique et quels types de réflexion seront menés dans l'action. » (Schön, 1983, p. 274) Le rôle choisi par un professionnel aura donc un impact important sur sa pratique : comme le dit Schön, «cela délimite l'envergure de l'intervention" (Schön, 1983, p. 274). Schön donne l'exemple d'un ingénieur : son rôle se limite-t-il à la résolution de problèmes techniques, ou doit-il aussi inclure la dimension relationnelle avec les clients? Des conflits peuvent ainsi surgir lorsqu'un professionnel envisage son rôle d'une façon différente de son institution, ou s'il estime que le cadre imposé est trop rigide. Dans le cas du jeu casual, le rôle des designers de jeux vidéo s'est révélé être un sujet problématique. En effet, alors même qu'il reste difficile d'expliquer en quoi consiste exactement l'activité d'un designer de jeux vidéo, celle-ci semble déjà être en pleine évolution.

\section{Collecte de l'expérience des designers de jeux}

15 Comme le savoir professionnel est tacite, il a été nécessaire de mettre en place des moyens de l'expliciter. Selon une approche qualitative, des rencontres ont été organisées avec huit designers de jeux de la région de Montréal et de Québec. Chaque rencontre a été précédée par la complétion d'un livret de sensibilisation destiné à stimuler la réflexion des participants.

16 L'échantillon est volontairement non probabiliste, et a été formé de façon déductive par rapport à la recension des écrits, ce que Pires définit comme étant un échantillon par homogénéisation: «il s'agit de prendre les informateurs les plus divers possible dans le groupe [ici les designers de jeux casual] afin de maximiser l'étude extensive du groupe choisi » (Pires, 1997, p. 71). Chaque designer a participé à la production d'au moins un jeu labellisé « casual » ayant été publié. Les huit participants appartiennent à cinq entreprises de jeux vidéo de taille variée, allant de moins de cinq employés à plus de deux mille. Les jeux ont été réalisés pour différentes plateformes: Nintendo Wii, Nintendo DS, Microsoft Kinect, Facebook ou encore des jeux Flash pour navigateurs Web et des jeux téléchargeables pour ordinateur personnel.

17 Avant la rencontre, les participants ont dû compléter un livret de sensibilisation sous forme de formulaire PDF. Il s'agit d'une application de la « sensitization » (Visser, 2005), terme traduit ici par «sensibilisation ». Le but est de sensibiliser les participants à un sujet afin de collecter des données plus étayées lors des rencontres. Ainsi, il a été demandé aux praticiens de se remémorer un projet de jeu casual en particulier afin d'être prêt à le détailler lors de l'entrevue. De plus, la sensibilisation permet de prendre du recul sur une pratique et encourage la réflexivité :

Durant la phase de sensibilisation, les participants effectuent une série de petits exercices conçus pour leur permettre de réfléchir à des expériences passées, et faire d'eux des "praticiens réflexifs". (Visser, 2005, p. 126, Schön, 1983).

18 Les livrets complétés ne sont pas voués à devenir des données à analyser. Ils sont des tremplins pour amorcer les entrevues, rendre la recherche attrayante et compréhensible aux yeux des participants.

19 Les rencontres proprement dites ont pris la forme d'entrevues semi-dirigées. Chaque designer a été invité à construire un "récit de pratique " (Goodman, Stolterman et Wakkary, 2011). Un tel récit consiste à rapporter, sous forme articulée et avec l'aide des questions du chercheur, les événements vécus au cours du développement d'un jeu 
vidéo. Il a été demandé aux participants de raconter leur expérience selon les différentes phases du projet, de la pré-production à la post-production. De telles entrevues ont semblé constituer un moyen simple et efficace d'accéder au savoir des designers. Mais cette méthode, lorsqu'elle est appliquée à l'industrie vidéoludique, soulève des difficultés cachées.

Pénétrer le monde des compagnies de jeux vidéo n'est pas une tâche aisée: le chercheur se heurte à la culture du secret de cette industrie. O'Donnell explique ainsi que cette culture se base sur une vision du développement de jeu comme étant une forme de magie noire, d'art occulte, voire de sorcellerie. Cette volonté de ne rien dévoiler apparaît tout d'abord au niveau corporatif avec la prolifération des accords de non-divulgation au sein des compagnies vidéoludiques (en anglais "non-disclosure agreement ", souvent abrégé en NDA). Il peut être très difficile pour le chercheur d'avoir accès à l'information, ou de rencontrer directement des designers de jeux, et encore plus difficile d'exploiter ce qui a été collecté. D'autre part, sans forcément entretenir activement l'opacité de leurs activités comme le ferait une société secrète, les designers de jeux vidéo tendent à conserver leur manière de faire des jeux vidéo dans une sorte de non-dit où rien n'est explicite, et à utiliser un jargon qui empêche le chercheur de saisir le sens de leur propos (O'Donnell, 2014).

21 Il semble nécessaire de revenir sur le rôle du chercheur pour comprendre ce qui a permis d'éviter ces obstacles dans cette recherche. En effet, l'accès à l'information et la compréhension du jargon n'ont pas été problématiques pour cette étude, du fait que la chercheure (c'est-à-dire l'auteure du présent texte) avait déjà participé, en tant que designer de jeux vidéo junior, à la production de plusieurs jeux vidéo dans un cadre professionnel. Dans une certaine mesure, on peut estimer que, sans posséder une expérience comparable à celle des participants, elle appartenait à leur monde, et était donc une initiée avec qui la discussion pouvait se faire sans traduction. Cela a grandement levé la barrière d'accès lié au jargon technique, et l'accès aux entreprises. Il est à noter que la chercheure ne connaissait personnellement aucun des participants, et que la méthode d'échantillonnage homogène a impliqué une recherche active de profils distincts. Tous les designers interrogés ont dû obtenir l'aval de leur compagnie avant l'entrevue, ce qui a été grandement facilité par les formulaires de consentement éthiques de l'Université de Montréal qui leur ont été présentés. Ces formulaires visent à protéger les participants d'une étude contre toutes conséquences néfastes. Dans ces formulaires, l'accent avait été mis sur l'anonymat, avec des règles plus rigides que celles du plus extrême des accords de non-divulgation. Cette garantie d'anonymat, combinée à la grande variété d'expérience des participants, a permis aux rencontres d'être très prolifiques. Loin des portraits lisses et sans accrocs que l'on lit parfois, les designers ont livré une description des problèmes et des « tabous » de leur milieu.

Les entrevues se sont alors transformées en un partage de connaissances entre praticiens, ce qui a facilité la collecte d'un savoir professionnel. Les participants ne sont pas simplement des informateurs, mais bien des partenaires avec qui il a été possible de construire une meilleure compréhension des phénomènes entrainés par l'avènement des jeux casual. Cet exercice s'est révélé extrêmement formateur. Pour la chercheurepraticienne, il s'agissait de conserver la spontanéité d'une conversation entre professionnels, tout en ne perdant pas de vue la nécessité d'obtenir des informations claires, précises et exploitables dans un contexte académique. Les moments de pure discussion entre praticiens produisent souvent un verbatim incompréhensible pour 
toute personne extérieure, composé d'amalgames de phrases inachevées et de références obscures. Les participants, quant à eux, devaient soudain prendre une place d'experts et rendre accessible leur savoir, faire preuve de pédagogie et se plier au jeu de la recherche, en particulier lorsque qu'ils étaient forcés de reformuler leur idée à de nombreuses reprises afin de produire des explications suffisamment limpides et compréhensibles pour un non-initié. Collecter le savoir professionnel de cette manière correspond à ce que Schön définit comme une réflexion sur l'action (1983, p. 278).

Les entrevues ont été entièrement retranscrites, puis analysées en utilisant le cadre théorique du praticien réflexif de Schön ${ }^{3}$. Les résultats ont ensuite été soumis aux participants afin d'écarter toute mécompréhension de leur propos. Les résultats présentés dans cet article concernent uniquement le rôle des designers de jeux: comment ce rôle se construit, se transforme, et quel impact il peut avoir sur la pratique, mais aussi sur la compréhension des évolutions vidéoludiques.

\section{Résultats : " réincarnation » du designer et construction d'un rôle d'agent d'évolution}

24 Le phénomène du jeu casual a fourni un contexte propice à la remise en question des pratiques de design. Par les obstacles que les praticiens ont rapportés dans leurs récits de pratiques, des stéréotypes concernant leur rôle ont progressivement été révélés. Les résultats présentés ici permettent d'explorer les liens entre l'évolution de la culture du jeu et l'évolution des pratiques de création.

25 L'analyse des propos des participants a conduit à la construction de différents rôles chez les designers de jeux vidéo : le «designer-joueur » et le "designer-mandataire ». Ces deux rôles vont être présentés, et vont permettre de comprendre en quoi les manières de faire des designers ont été remises en question. Chacun de ces rôles a un impact sur la pratique du design de jeux vidéo et sur l'évolution des jeux.

\section{Le stéréotype du » designer-joueur hardcore »}

Il peut être difficile de comprendre comment se construit le rôle d'un professionnel. Cependant, pour les participants de cette étude, il est ressorti très nettement que leur rôle de designer était fondé sur un autre rôle, antérieur: celui de joueur. Chaque praticien, avant d'être un designer de jeux, est un joueur.

L'expérience des designers en tant que joueurs est un socle pour justifier leurs aptitudes de design. En effet, les activités de design de jeux vidéo demeurent peu claires, et il reste beaucoup à faire pour théoriser les outils et les concepts des designers de jeux vidéo. ${ }^{4}$ Les designers n'ont pas attendu que la recherche leur fournisse un vocabulaire pour développer leur pratique. Ils ont élaboré leurs propres stratégies. L'une d'elles consiste à s'appuyer sur le seul élément qu'ils partagent avec toute l'équipe de production: les jeux. O'Donnell appelle cette façon d'utiliser les jeux comme vecteurs de connaissance le "game talk», que l'on peut traduire par "discours par les jeux »: nommer un jeu permet de faire référence au système dudit jeu, à ses mécaniques, ainsi qu'à l'expérience qu'il fait vivre au joueur. De ce fait, le profil de joueur de ces designers, leurs goûts et leurs expériences sont une base pour discourir par les jeux, et constitue une assise pour leur pratique. 
28 Le terme "designer-joueur » désigne alors un rôle de designer de jeux construit principalement autour de cette expérience de joueur. Les designers cherchent à aligner leur pratique avec leurs valeurs en tant que joueurs et utilisent ainsi leurs habitudes de jeu comme étalon pour juger de la qualité de leur projet.

29 Le rôle du designer-joueur semble rattaché à une vision traditionaliste du designer de jeux vidéo comme joueur hardcore. Ainsi, dans les entrevues réalisées par Jesper Juul avec des designers de jeux casual, on peut lire cet extrait :

30 Les développeurs de jeux hardcore font des jeux pour eux-mêmes («J'aime ça mettons-le dans le jeu »), tandis que les développeurs de jeux casual font des jeux pour eux-mêmes et le reste du monde ("J'aime ça mais vérifions que cela fonctionne aussi avec mon père/ma sœur/ma réceptionniste ») (Juul, 2009, p. 76).

31 Cette citation montre qu'avec l'apparition de jeux casual, le designer doit changer son approche. Il semble implicitement admis que les goûts du designer en tant que joueur sont différents de ceux du public casual, car le designer est un joueur hardcore. Lors des entrevues, cette assomption d'un designer-joueur hardcore était présente chez la plupart des participants : il leur semblait évident qu'ils ne jouaient pas à des jeux casual pour leur plaisir. Ils ont ainsi déclaré :

Du point de vue du designer, le sentiment principal qu'on a c'est qu'on ne fera pas les jeux auxquels on a envie de jouer. [Les designers] veulent faire les jeux auxquels ils veulent jouer (Participant 1 ).

Je ne crois pas qu'il y en ait beaucoup [de designers de jeux] qui font des parties de Farmville... (Participant 3).

Aimez-vous le jeu que vous avez obtenu? Oui ! [...] en tant que joueur jamais je ne vais y jouer. Mais je suis très content de ce que j'ai fait par contre (Participant 8).

Ces designers décrivent la disjonction qu'ils ont constatée entre leurs goûts en tant que joueurs et les goûts de leur public cible. Ils affirment ne pas être de grands adeptes des jeux casual et estiment que ce n'est pas non plus le cas de leurs collègues. Ces designers vivent le jeu casual comme une " intrusion ", comme l'apparition d'un nouveau style de jeux dans lequel ils ne se reconnaissent pas. L'un d'eux souligne: " dans mon cas, j'ai fait en tout deux jeux casual, et je suis sorti du genre» (Participant 3). Leurs perceptions à l'égard du jeu casual sont celles de la rupture décrite par Juul, d'une révolution à laquelle ils doivent prendre part malgré eux. Ils n'estiment pas être à l'origine de ce style de jeux : leur direction leur a imposé ce type de projet.

L'apparition d'un nouveau style de jeu semble ici avoir permis de révéler l'importance que les designers accordent à leur rôle de joueur, et le stéréotype que celui-ci semble impliquer: le designer de jeux vidéo est un joueur hardcore. Pourtant, d'autres entrevues montrent que ce premier stéréotype est déjà en voie d'être dépassé.

\section{Dépasser le stéréotype hardcore : le « designer-joueur casual »}

Bien que de nombreux participants aient, au premier abord, donné l'impression que le designer de jeux vidéo est un joueur hardcore, d'autres entrevues sont allées à l'encontre de cette assomption : le rôle du designer-joueur évolue. De plus, l'idée d'un rôle de designer construit uniquement par rapport à une expérience de joueur possède certaines limites.

Tout d'abord, certains participants ont écarté d'emblée l'idée qu'ils pouvaient être désignés comme des joueurs hardcore : 
J'ai pas énormément de temps non plus donc je suis plus tenté par le casual [le fait de jouer à des jeux casual], donc c'est pour ça que je crée des jeux casual en partant (Participant 5).

J'ai beaucoup moins de temps pour rentrer dans un jeu ou juste commencer un nouveau jeu [...] Fait que souvent je joue juste à des jeux casual.[...] Je dis ok, sur l'heure du midi, si ça me prend mes deux mains je jouerai pas au jeu [rires]! (Participant 6). jeux casual. Pour le participant 6, il semble naturel de jouer tout en mangeant: on retrouve l'interpénétration des activités constatée par Kultima. De plus, il semble totalement décomplexé à l'idée de jouer d'une seule main, et il ne semble pas nécessaire pour lui d'être un joueur hardcore pour construire son rôle de designer. Ces deux derniers designers n'ont jamais participé à la création de jeux pour un public dit hardcore. Ils sont arrivés dans le monde du jeu vidéo par une formation en multimédia axée sur le Web. Progressivement, en participant à des compétitions de programmation et en s'initiant à des outils comme Adobe Flash, ils en sont venus à créer des jeux vidéo. Leurs entreprises ont d'ailleurs été créées plus récemment que celles des autres participants. Leur parcours en tant que créateur de jeux s'inscrit dans l'optique de normalisation de la culture numérique (Kultima, 2009). Ils n'ont pas vécu de fracture au sein de leur pratique comme cela a pu être le cas des participants évoqués précédemment.

Ces résultats montrent que l'association entre joueurs hardcore et designers de jeux vidéo est en train de s'effriter. Avec le témoignage de designers appréciant les jeux casual, il semble soudainement bien réducteur d'avoir pu penser que tous les designers étaient des joueurs hardcore.

38 De plus, ces designers d'un nouveau genre permettent de remettre en perspective les explications d'évolution de la culture du jeu présentée dans la première partie de cet article. En effet, dans les études sur les jeux vidéo casual, la naissance et l'expansion de ce type de jeux semblent toujours traitées comme un «phénomène » qui s'est réalisé de lui-même. Il est rarement question des aspirations des designers qui sont à l'origine desdits jeux casual. Leur rôle est vu comme primordial, comme en témoigne le fait que les chercheurs les interrogent, mais l'image du designer de jeux adepte de jeux hardcore semble toujours présente en filigrane. Les designers choisis dans les études sont souvent très expérimentés et ont majoritairement travaillé sur des jeux hardcore. L'échantillon par homogénéisation a permis de montrer l'existence d'un autre rôle du designer, bien moins lié à la culture hardcore. Ces designers viennent détruire le mythe d'une génération spontanée du jeu casual: les jeux casual seraient apparus soudainement, et l'industrie semble avoir dû suivre une machine déjà lancée. Les participants de cette étude montrent qu'il existe des créateurs qui ont initié ce changement, car ils font eux-mêmes partie de ces nouveaux joueurs.

Ce constat mène cependant à un autre questionnement : les designers jouant à des jeux casual sont-ils les seuls aptes à créer des jeux casual?

\section{Le « designer-joueur instrumental »}

Le rôle de designer-joueur hardcore semble presque handicapant sur les projets de jeux casual. Si la construction du rôle d'un designer se fait avant tout par rapport à son expérience de joueur, alors son expertise s'en trouve limitée. C'est malheureusement 
une vision à laquelle se sont heurtés plusieurs participants. Ainsi le Participant 1 , reconnu pour son expertise en développement de jeux vidéo hardcore et son rôle de joueur hardcore, a présenté à son équipe une version de démonstration d'un jeu casual ; le jeu a été jugé beaucoup trop difficile, et l'équipe a estimé que le participant ne comprenait pas le jeu casual :

L'erreur en développement que j'avais faite, c'est que j'avais commencé par construire le dernier niveau, où tous les ingrédients sont présents, où toute la complexité est là. Mais quand on le faisait essayer aux gens, incluant le président, c'était beaucoup trop épeurant comme expérience! Il a dit « Ah non, non, ça peut pas être ça ! ». Et la confiance avait été perdue sur le jeu et son potentiel, parce qu'il avait l'impression qu'on ne faisait pas un jeu casual. [...]

41 Le participant 8 a décrit une expérience similaire; lorsqu'il a introduit des niveaux d'une difficulté plus avancée dans son jeu, son supérieur a estimé qu'il se basait sur ses propres attentes de joueur hardcore, et non sur celles d'un public casual :

Les personnes sur le projet vont jouer les classes de danse rapides pour voir ce que c'est, puis là elles sont comme : «C'est trop difficile, c'est trop difficile !».

Ces participants ont vu leur équipe réduire leur rôle de designer à celui de joueur : le design ne serait qu'un transfert de leurs goûts personnels et les designers seraient condamnés à se cantonner à un style de jeux. Les participants ont fermement renié cette vision du design et ont souligné le fait qu'ils sont capables de mettre en perspective leur rôle de joueur :

C'est une autre attitude qu'il faut prendre quand on critique les choses, c'est qu'il faut les voir pour un public en particulier, et non pour critiquer en tant que « moi ce que je joue dans mes habitudes de vie » (Participant 8).

Selon les designers interrogés, leur rôle de joueur est important pour leur pratique, mais il doit être vu comme ce que O'Donnell appelle un rôle «instrumental »: «A la place, je propose un autre terme, joueur instrumental, qui s'applique à des joueurs qui ne sont pas focalisés sur un genre en particulier ni sur un type de jeu, mais qui tentent systématiquement et invariablement de disséquer les jeux pour en révéler les mécaniques » (p.63). Le designer de jeux est bien un joueur, et disséquer, analyser et comprendre des jeux est une facette majeure de sa pratique; néanmoins, il effectue ces activités en se détachant de son profil de joueur. Le participant 3 décrit cette façon « instrumentale » de jouer avec la métaphore d'un marionnettiste :

Tu veux y jouer pour essayer, tu vas passer peut-être une semaine, question de voir ce que c'est exactement, mais après ça tu comprends le principe, tu vois les cordes immenses en arrière qui tirent, un peu comme les marionnettes...

Pour la majorité de nos participants, cette dimension instrumentale était renforcée par le manque de possibilités de mener des tests auprès de leur public cible. En effet, en raison de budgets souvent limités, aucun test externe, avec des joueurs recrutés pour l'occasion, n'a été effectué ; seuls des tests internes, avec différents volontaires au sein de l'entreprise, ont pu être conduits. De ce fait, la capacité des designers à mener une pratique instrumentale était d'autant plus cruciale. Le participant 7 résume bien la difficulté d'une telle situation :

Souvent on sort nos jeux avec notre « guts feeling " parce qu'on ne prend pas assez le temps d'aller chercher le public cible. On fait beaucoup de jeux pour les enfants et pour les femmes puis on est bourré d'hommes [rires].

Pour certains des participants, les projets de jeux casual ont donc posé de véritables problèmes de pratique: leurs capacités de design ont été remises en question et la 
confiance qu'on leur accordait a été perdue. Malgré tout, ces designers, face à l'adversité, ont réussi à se réinventer et ont endossé un nouveau rôle: celui de « designer-mandataire».

\section{Dépasser le stéréotype du « designer-joueur » : le « designer- mandataire »}

Comme souligné précédemment, un grand nombre de designers semblent demeurer des adeptes de jeux hardcore et préfèrent travailler sur ce style de jeux. Pour autant, face à une forte demande, ils ont bien souvent été obligés de créer des jeux casual. Ils ont alors été capables de s'éloigner fortement du rôle de designer-joueur pour se transformer en « designer-mandataire » :

Le défi de design est toujours : atteindre l'objectif qui est visé par le mandat. [...] Ce jeu-là, à l'interne, a atteint les objectifs visés. Il a respecté tous les mandats qui avaient été demandés (Participant 3).

Le designer-mandataire correspond aux designers estimant que leur rôle consiste à répondre à un mandat, selon le bon vouloir du mandant (par exemple le producteur), peu importe leur rôle de joueur. Les participants ont souligné que la capacité à devenir un "mandataire du fun» pour un public aux goûts différents des leurs est une expertise qui fait partie de leur rôle. Leur rôle de designer ne se construit plus sur leur rôle de joueur; au contraire le rôle de designer est primordial, et il a préséance sur celui de joueur. La dimension du jeu est alors bel et bien traitée de façon instrumentale.

En l'état, ce rôle du designer-mandataire n'apporte que peu d'éléments supplémentaires à l'analyse du jeu instrumental de O'Donnell. Mais les témoignages recueillis montrent que les implications de cette position de mandataire vont plus loin qu'une simple capacité à instrumentaliser le jeu. Les manières de faire, les façons de travailler, les outils de communications des designers ont été remis en question, et ce sont les designers eux-mêmes qui ont mené un processus de changement pour les améliorer.

Ainsi, face aux jeux casual, les designers ont questionné leurs pratiques, qui s'appuyaient peut-être démesurément sur le stéréotype du designer-joueur. Le participant 7 explique ainsi qu'il souhaiterait réaliser des études plus poussées de différents publics, car analyser les jeux déjà sortis n'est pas suffisant :

Donc des fois on explique notre design en disant «tel autre jeu a marché pour les femmes, celui-là va marcher parce qu'il a des mécaniques similaires », mais on n'en sait rien parce qu'on l'a jamais mis dans les mains d'une femme puis on n'a pas le temps de vérifier ! C'est risqué...

50 De nouvelles méthodes de travail sont alors proposées par les designers. Le participant 1, qui avait l'habitude de montrer le dernier niveau d'un jeu lors des séances de démonstration en interne, présente désormais le jeu différemment :

Si tu développes un jeu casual, la première version interactive que tu fais du jeu doit correspondre au premier niveau de jeu, ou à la première expérience de jeu que quelqu'un va vivre [...]. L'attente c'est de démontrer l'expérience de jeu, qui est casual, donc qui remplit tous les besoins au niveau de l'apprentissage, qui dès le départ est fun, etc.

51 Ce participant a aussi arrêté de produire d'énormes documents de design. Habituellement, le document de design (en anglais game design document ou GDD), réalisé principalement par le designer, est la bible du jeu, il contient tous les détails et 
sert de guide à toute l'équipe, et peut atteindre des milliers de pages. Pour les jeux casual, un tel document semblait moins adapté :

Au niveau du document de design, si c'est un haut niveau de risque conceptuel, on fait des documents d'au maximum une page, où la plupart de la conversation c'est juste une image, et on fait des itérations. La formule idéale pour moi c'est trente minutes, où on a deux ou trois propositions, on discute, et on voit ce que les gens aiment ou pas (Participant 1).

Dans ce dernier témoignage, la pratique a été rendue plus transparente et plus ouverte à des avis divers. Le jeu casual semble permettre une plus grande inclusion au sein même des entreprises et non uniquement au sein des communautés de joueurs.

Ces exemples de modifications des pratiques viennent illustrer la réflexion générale sur leur rôle qu'ont menée les designers de jeux vidéo confrontés à la création d'un jeu casual. Certains d'entre eux ont vécu une prise de conscience :

Pour moi qui n'avais pas beaucoup d'expérience, un jeu casual était une manière de faire de l'argent. On sort des petits jeux, des mécaniques simples, mais fun, et puis on met ça dans une boite, point d'interrogation, profit! [En fait] il faut que ce soit fait sérieusement, ça demande une réflexion derrière, et n'importe qui ne peut pas s'improviser créateur de jeux casual (Participant 3).

Pour ces participants, être capable d'analyser des jeux casual n'est pas suffisant pour être un bon designer: le rôle de designer ne se résume pas à être un bon joueur instrumental. Un designer doit pouvoir prendre du recul sur les jeux, mais aussi sur ses pratiques et sur tout un système de production :

[...] je me suis questionné, je me suis challengé moi-même, j'ai... J'ai essayé, avant de proposer. [...] Il faut travailler fort, il faut se questionner, il faut même se faire chier, se remettre en question avant d'arriver à quelque chose qui se tient vraiment (Participant 8).

55 Le rôle du designer-joueur apparaît trop limitatif, la pratique d'un designer ne se résume pas à parler et analyser des jeux, mais comprend bien d'autres activités. La capacité des designers à créer de nouveaux systèmes, à s'intégrer au sein d'une équipe de production ou à modifier des pratiques sont des activités qui viennent témoigner de l'ampleur du rôle des designers de jeux. Les conseils trouvés sur les sites Web professionnels tendent à corroborer cette vision, avec des articles insistant sur les compétences « hors-jeu » dont doivent faire preuve les designers de jeux vidéo, tels que la capacité à communiquer clairement et efficacement (Clark, 2014).

Cette remise en question des pratiques est conduite dans un esprit d'amélioration du paysage vidéoludique. Par leur réflexion et leur travail, les designers interrogés espèrent influencer la qualité des jeux produits et contribuer à l'évolution des cultures du jeu. Un des participants a ainsi suggéré qu'il manquait d'expérience dans le domaine casual, et regrette de ne pas avoir réussi à "marquer l'histoire du jeu», tandis qu'un autre s'est intéressé aux jeux de danse et a souligné la minutie dont il faut faire preuve pour leur conception. La complexité du processus de design semble devenir un sujet majeur pour les designers soucieux d'améliorer leur pratique. En ce sens, nos résultats rejoignent l'étude d'Audureau lorsque celui-ci déclare à propos de Miyamoto :

[... ] c'est un vrai professionnel au sens le plus large du terme. C'est quelqu'un qui a des méthodes de travail très réfléchies, qui fait énormément de veille industrielle, qui a des processus de création qui sont en partie artisanaux mais en même temps très pensés (Verlet, 2014). 

de jeux de celle du design en général : design architectural, design d'intérieur, design industriel ou encore design graphique, dont les problématiques sont semblables à celles du design de jeux. En effet, à leurs débuts, les tenants de ces disciplines étaient eux aussi vus comme des «magiciens» (Jones, 1970, p. 46). De plus, tout comme les études $d u$ jeu semblent excessivement centrées sur les jeux, les théories en design ont longtemps été focalisées sur l'objet réalisé, tel que le bâtiment dans le cas de l'architecture. Cependant, Findeli et Bousbaci (2005) ont montré qu'il s'était produit peu à peu une " éclipse de l'objet »: les théories en design se sont progressivement intéressées au processus de conception (ses étapes, sa logique), puis aux acteurs et aux parties prenantes du projet, laissant de côté l'objet. Le récent article «L'Apport des théories du design aux game studies : nouvelles perspectives en design de jeux vidéo " (Chiapello, à paraitre en 2015) montre qu'une évolution semblable se profile dans les théories en design de jeux et qu'un rapprochement entre design de jeux et design en général est donc possible. Ce rapprochement peut se faire autour de la façon de mener le processus de design, et l'on pensera alors à des étapes de conception communes, telles que l'analyse des besoins ou l'établissement du cahier des charges. Mais des parallèles plus innovants sont aussi envisageables: les modèles les plus récents en design sont centrés sur les acteurs, en particulier leurs valeurs, leurs expériences et leur façon de voir le projet, en un mot leur éthique. La présente réflexion sur le rôle des designers de jeux s'inscrit dans cette dernière vague de théories. champ comme celui du design n'est pas la simple production de connaissances, mais la pédagogie; révéler les modèles sous-jacents d'une discipline permet aussi de s'en distancier, pour éventuellement les transformer et les bonifier. Dans cette optique, on peut se questionner sur la genèse des préconceptions entourant le rôle de designers de jeux vidéo. De nombreux programmes universitaires ont aujourd'hui pour but la formation de designers de jeux (Entertainment Software Association, 2014) : quelle est la vision du designer, et du design en général, communiquée aux étudiants? Les programmes sont-ils conçus en prenant en compte une dimension éthique? Selon Schön, seul un designer conscient du rôle dans lequel il se meut sera à même d'en pallier les faiblesses; une formation permettant la réflexivité constitue alors un enjeu majeur pour l'amélioration des pratiques.

Dans le cadre de cet article, ne pas limiter les designers de jeux à des designers-joueurs permet de leur reconnaître des capacités d'agents d'évolution importantes : par leur réflexivité, les designers interrogés ont montré qu'ils étaient capables de s'adapter à un nouveau style de jeux, et qu'ils pouvaient s'en servir comme opportunité pour modifier leurs habitudes.

61 Cette recherche suggère ainsi un mécanisme de coévolution des produits et des pratiques : tel un cercle vertueux, une nouvelle façon d'envisager les jeux - ici les jeux casual - peut provoquer une évolution des pratiques de design, évolution susceptible à son tour de déboucher sur une nouvelle vague d'œuvres venant transformer le paysage vidéoludique, remettant en question certaines pratiques de design, et ainsi de suite. Dans le cadre de cette évolution, joueurs et designers sont tout aussi importants et 
fortement liés : l'évolution ne peut se faire de façon désincarnée. Cependant, leur lien n'est pas simplement celui contenu dans le rôle de designer-joueur : le designer va vers de nouveaux publics et peut contribuer à renouveler les pratiques de l'industrie de l'intérieur pour amener plus de diversité.

\section{Conclusion}

62 Cet article a essayé de montrer l'importance et la complexité rattachées à la définition du rôle du designer de jeux vidéo. La rareté des études concernant les pratiques de design de jeux mène à un amalgame de stéréotypes qui méritent d'être plus minutieusement examinés. Les résultats suggèrent que les designers de jeux vidéo sont des agents d'évolution de l'industrie vidéoludique et des jeux en eux-mêmes, à condition de ne pas les enfermer dans des rôles réducteurs.

Pour les designers dont le rôle était encadré par leur expérience de joueur hardcore, l'arrivée du jeu casual a pu être vécue comme une révolution : ils ont dû réinventer leur rôle. Pour ceux qui n'étaient pas rattachés à la culture hardcore, le design de jeux casual correspond plutôt au modèle de la normalisation du numérique de Kultima. Ils sont pourtant parfois à l'origine même des changements, car ils ont pénétré le monde fermé des designers-joueurs hardcore, et leurs œuvres ont provoqué la transformation du paysage vidéoludique.

64 Les expériences divergentes de ces designers viennent remettre en perspectives les modèles proposés par Juul et par Kultima, et montrent en quoi ils peuvent être vus comme complémentaires.

La façon d'envisager le rôle d'un designer peut aussi limiter notre compréhension de l'évolution de l'histoire du jeu, en écartant le point de vue des designers. Si le designer est vu uniquement comme un joueur, la richesse de son expérience est passée sous silence. Un designer-joueur semble enfermé dans son univers : il ne serait capable de travailler qu'avec les jeux auxquels il joue, restant confiné dans un certain genre. La rareté des études concernant les pratiques de design de jeux explique peut-être la formation de ce stéréotype. La présente recherche a voulu pallier ce manque et montrer que les designers de jeux vidéo ne sont pas uniquement des joueurs : une partie de leur rôle consiste bien à jouer, mais il s'agit d'une pratique instrumentale du jeu et elle n'est pas l'unique activité de cette profession. Les capacités à penser les outils, les processus de communication et les manières de faire sont tout aussi importantes que les capacités d'analyse des jeux vidéo. Ignorer ces éléments empêche de saisir pleinement le rôle des designers dans l'évolution des jeux. Les accepter permet de voir le designer comme un mandataire, capable de faire le lien entre des jeux et des publics variés, et comme un puissant agent de changement pour les jeux comme pour l'industrie. Les designers sont alors une forme d'incarnation du changement, et les études de la pratique du design de jeux vidéo semblent alors plus que jamais nécessaires pour appréhender l'évolution vidéoludique dans son ensemble. 


\section{BIBLIOGRAPHIE}

AUDUREAU W. (2014), Sur les traces de Shigeru Miyamoto, Cergy, Pix'n Love.

CHIAPELLO L. (à venir en 2015), «L'Apport des théories du design aux game studies : nouvelles perspectives en design de jeux vidéo ", RACAR Revue d'art Canadienne / Canadian Art Review, Numéro spécial : Les études du design au Canada et au-delà : un état des lieux.

CHIAPELLO L. (2012a), Les casual games : définition à l'aide du savoir professionnel des designers de jeux, mémoire de maîtrise, Faculté de l'aménagement, Université de Montréal.

CHIAPELlo L. (2012b), « Utiliser les travaux de Donald Schön pour étudier les jeux vidéo : exemple de la définition du casual game». Actes du deuxième colloque de la recherche en aménagement « Diversités et Convergences », Montréal, 2012, p. 23-38.

CLARK O. (2015), « What makes a good game designer ? », Develop, http://www.develop-online.net/ opinions/what-makes-a-good-game-designer/0199138, consulté le 25 juin 2015.

COSTIKYAN G. (1994), « I Have No Words \& I Must Design », Interactive Fantasy, vol. 2, n 2, pp. 9-33, en ligne au http://www.costik.com/nowords.html.

DJAOUTI D., ALVAREZ J. et JESSEL J.-P. (2010), « Concevoir l'interactivité ludique : une vue d'ensemble des méthodologies de « Game Design », Colloque Ludovia 2010 (Ariège-Pyrénées)

DONOVAN T. (2010), Replay: The history of video games, Lewes, UK, Yellow Ant.

ENTERTAINMENT SOFTWARE ASSOCIATION (2014), Essential Facts About The Computer and Video Game Industry, en ligne: http://theesa.ca/wp-content/uploads/2014/11/ESAC-Essential-

Facts-2014.pdf .

FINDELI A. et BOUSBACI R. (2005), «L'Eclipse de L'Objet dans les Théories du Projet en Design », EAD 06 (Brême, Allemagne), en ligne au http://projekt.unimes.fr/files/2014/04/Findeli. 2005.Eclipse.objet_.pdf. GOODMAN E., STOLTERMAN E. et WAKKARY R. (2011), « Understanding interaction design practices », Annual Conference on Human Factors in Computing Systems, Vancouver, BC, Canada. ICHBIAH D. (2013), Eric Chahi : parcours d'un créateur de jeux vidéo français, Cergy, Pix'n Love. JONES J. C. (1970), Design Methods: seeds of human futures, New York, John Wiley \& Sons. JUUL J. (2009), A Casual Revolution Reinventing Video Games and Their Players, Cambridge, The MIT Press.

KUITTINEN J. et HOLOPAINEN J. (2009), « Some notes on the nature of game design », DiGRA 2009 : Breaking New Ground : Innovation in Games, Play, Practice and Theory (Brunel University, Londres).

KUitTinen J., KULTIMA J. A., NIEMEL J. et J. PAAVILAINEN (2007), « Casual Games Discussion », Colloque Future Play 2007 (Toronto, Canada)

KULTimA A. (2009), « Casual game design values », $13^{\circ}$ colloque international MindTrek: Everyday Life in the Ubiquitous Era (Tampere, Finland).

KULTIMA A.,KÖÖNIKKÄ J. et KARVINEN J. (2011), « The Four Different Innovation Philosophies Guiding the Game Development Processes », Séminaire de recherche Games and Innovation 2011 (Tampere). 
MAKINO T. et GORGES F. (2010), Gunpei Yokoi : Vie \& Philosophie du Dieu des jouets Nintendo, Cergy, Pix'n Love.

MALABY T. M. (2009), Making virtual worlds : Linden Lab and Second Life, Londres, Cornell University

Press.

o'DonNELL C. (2014), Developer's Dilemma The Secret World of Videogame Creators, Cambridge, The MIT press.

PIRES A. (1997), «Échantillonnage et recherche qualitative : essai théorique et méthodologique », dans J. Poupart (dir.), La recherche qualitative: Enjeux épistémologiques et méthodologiques, Boucherville, Gaëtan Morin, pp. 86-109.

scHÖN D. (1983), The Reflective Practitioner : How Professionals Think in Action, New York, Basic Books.

SCHÜLL N. D. (2012), Addiction by design : Machine gambling in Las Vegas, New Jersey, Princeton University Press.

TREFRY G. (2010), Casual Game Design Designing Play for the Gamer in All of Us, Elsevier Inc.

VERLET N. (2015), « Interview : « Sur les traces de Miyamoto » », Gamekult, http://

www.gamekult.com/actu/interview-sur-les-traces-de-miyamoto-A134913.html.

VISSER F. S., STAPPERS P. J., VAN DER LUGT R. et SANDERS E. (2005), « Contextmapping :

experiences from practice », CoDesign International Journal of CoCreation in Design and the Arts, vol. 1, $\mathrm{n}^{\circ} 2$, pp. 119-149.

WONDRA G. (2015) « Death of the Game Designer », Gamasutra.com, http://www.gamasutra.com/

blogs/GregWondra/20150209/235998/Death_of_the_Game_Designer.php

\section{NOTES}

1. Du fait de la complexité du phénomène qu'ils désignent dans le domaine vidéoludique, le terme «casual » et son prétendu opposé « hardcore » demeurent difficiles à traduire. Le terme hardcore désigne le plus souvent des jeux demandant un fort investissement, un engagement passionnel venant de joueurs invétérés. Ces caractéristiques ont tout d'abord été vues comme étant opposées à celles des joueurs casual. Les dictionnaires proposent ainsi généralement deux équivalents pour le terme casual : le jeu « occasionnel » ou le jeu « décontracté ». Cependant, les études ont montré que cette distinction ne fonctionne pas. Les joueurs casual sont souvent assidus et passionnés (Kuittinen et al., 2007), et se montrent prêts à recommencer une partie de nombreuses fois afin de gagner (Juul, 2009). Dans ces conditions, il semble difficile d'utiliser les traductions actuellement proposées et nous préférons garder les termes anglais.

2. Les citations en anglais ont été traduites en français par l'auteure.

3. Pour plus d'information sur le cadre théorique utilisé, le lecteur peut se référer à Chiapello (2012b).

4. Voir le texte I Have No Words \& I Must Design (Costikyan, 1994) 


\section{RÉSUMÉS}

Cet article propose de montrer l'importance des designers de jeux vidéo en tant qu'agents d'évolution des jeux. Il s'appuie sur une étude dont le but principal est de mieux définir les jeux dits « casual » en utilisant le savoir professionnel des designers de jeux vidéo. En documentant ce sujet, il est apparu que les pratiques des designers de jeux sont en pleine évolution. Étant donné le peu d'informations existantes sur le rôle de ces designers dans la littérature scientifique, il a semblé opportun d'approfondir ce sujet. Les résultats montrent la présence d'un phénomène intrigant: les problématiques liées au jeu casual semblent avoir un impact sur la façon d'envisager le rôle des designers de jeux. Or, ce rôle est déterminant pour notre compréhension de l'évolution des jeux vidéo.

This article aims to demonstrate the importance of video game designers as agent of progress. This work is based on a study whose main purpose is to better define casual games while using the video game designers' professional knowledge. During the course of the study, it became clear that the game design practices have been evolving over time. Due to the scarcity of existing information on the role of designers in the scientific literature, it seemed appropriate to explore this lack. The results show the presence of an intriguing phenomenon: the problems related to casual games seem to have a direct impact on the role the designers play; and this role is critical to our understanding of the evolution of video games.

\section{INDEX}

Keywords : video game design, design practice, designer role, professional knowledge, instrumental player, casual game

Mots-clés : design de jeux vidéo, pratique du design, rôle du designer, savoir professionnel, joueur instrumental, jeu casual

\section{AUTEUR}

\section{LAURELINE CHIAPELLO}

Université de Montréal 